Pathologe 2008 · [Suppl 2] 29:223-231

DOI 10.1007/s00292-008-1025-z

Online publiziert: 28 . September 2008

c) Springer Medizin Verlag 2008

\author{
G. Jundt ${ }^{1,2} \cdot$ D. Baumhoer ${ }^{2}$ \\ ${ }^{1}$ Knochentumor-Referenzzentrum, Institut für Pathologie \\ des Universitätsspitals Basel, Schweiz \\ ${ }^{2}$ Institut für Pathologie, Universitätsspital Basel, Schweiz
}

\title{
Chondrogene Tumoren des Skeletts
}

Knorpeltumoren gehören mit einem Anteil von etwa $36 \%$ zu den häufigsten im Register des Basler Knochentumor-Referenzzentrums (KTRZ) erfassten primären Knochentumoren (• Tab. 1). Sie repräsentieren die Mehrzahl der gutartigen Tumoren und stehen nach dem Osteosarkom an zweiter Stelle der bösartigen Knochentumoren.

Während die weit überwiegende Mehrzahl der Chondrosarkome eher Tumoren des 4.-7. Lebensjahrzehnts sind, kommen gutartige Knorpeltumoren meist bei Jugendlichen und jungen Erwachsenen vor (2.-3. Lebensjahrzehnt) [9].

Gutartige Knorpeltumoren umfassen die sehr häufigen Osteochondrome und Chondrome (intraossäre Enchondrome und oberflächliche periostale Chondrome) sowie die wesentlich selteneren Chondroblastome und Chondromyxoidfibrome. Sie unterscheiden sich jedoch voneinander nicht nur in ihrer Morphologie, sondern auch bezüglich ihrer Topographie im Knochen (epiphysär, metaphysär, diaphysär) und ihrer Lokalisation im Skelett (lange oder kurze Röhrenknochen, flache Knochen).

Ähnliches gilt auch für die malignen Knorpeltumoren, die histologisch in die häufigen klassischen Chondrosarkome (Grad 1-3) und die weit selteneren klarzelligen, periostalen, dedifferenzierten und mesenchymalen Chondrosarkome unterteilt werden können. Auch sie haben topographische und radiologische Charakteristika, die in der Differenzialdiagnose helfen.

Widmung. Herrn Prof. Dr. A. Schulz zum 65. Geburtstag gewidmet.
Da Knorpelgewebe zu Verkalkungen oder metaplastischen Verknöcherungen neigt, die dem eher lobulären Muster der Tumorformationen folgen und meist deren Peripherie betreffen, lassen sich radiologisch im konventionellen Röntgenbild typische bogenförmige oder kreisförmige Verschattungsmuster erkennen [9].

Je nach Wachstumsgeschwindigkeit eines Tumors wird der ortsständige Knochen unterschiedlich schnell destruiert, sodass dem Knochen unterschiedlich viel Zeit bleibt, auf die Alteration zu reagieren: Langsam wachsende (meist gutartige) Tumoren, wie z. B. das Chondroblastom, induzieren einen Sklerosesaum, der bei schnell wachsenden (meist bösartigen Läsionen) fehlt oder nur unvollkommen ausgebildet ist. Auch wenn bereits das konventionelle Röntgenbild dem Pathologen wertvolle Informationen liefert und ihm helfen kann, die morphologischen Befunde der oft nur als Fragmente vorliegenden Biopsiepartikel richtig einzuordnen, empfiehlt es sich, zumindest in Zweifelsfällen, für Diagnose und Therapieplanung ein Referenzzentrum einzuschalten, da die Histologie der verschiedenen Knorpeltumoren gelegentlich große Überlappungen auch mit nichtkartilaginären Tumoren aufweisen kann. Die Auswirkungen von klinisch-diagnostischer Kompetenz auf Verlauf und Prognose ossärer Tumoren ist mehrfach belegt $[2,13]$.

Molekulargenetische und immunhistochemische Untersuchungen haben zwar viel zum Verständnis der Entwicklung von Knorpeltumoren beigetragen [23], jedoch nur in wenigen Fällen Eingang in den diagnostischen Alltag gefunden, weshalb auf ihre Darstellung weitgehend verzichtet wird.

\section{Osteochondrome und multiple kartilaginäre Exostosen}

Osteochondrome wurden lange Zeit als entwicklungsbedingte Fehlbildungen angesehen. Neuere genetische und molekulargenetische Untersuchungen sprechen jedoch dafür, dass es echte Tumoren sind [10]. Mit etwas über $35 \%$ sind sie im Material des KTRZ Basel die häufigsten gutartigen Knochentumoren (m.: w. = 2:1), wobei ihre tatsächliche Zahl sicher höher liegt, da sie meist keine Symptome verursachen und deshalb nicht diagnostiziert werden. Man kann breitbasige (sessile) von gestielten (pedunkulierten) Osteochondromen unterscheiden. Beide besitzen eine Knorpelkappe, deren Aufbau den der Epiphysenfuge nachahmt. Säu-

\begin{tabular}{|c|c|}
\hline Tumortyp & Anzahl \\
\hline Maligne & 547 \\
\hline $\begin{array}{l}\text { Chondrosarkom (inklusive sekun- } \\
\text { däres Chondrosarkom; } n=12 \text { ) }\end{array}$ & 437 \\
\hline Dedifferenziertes Chondrosarkom & 45 \\
\hline Klarzellchondrosarkom & 21 \\
\hline Periostales Chondrosarkom & 25 \\
\hline Mesenchymale Chondrosarkom & 19 \\
\hline Benigne & 1582 \\
\hline $\begin{array}{l}\text { Osteochondrom (inklusive } \\
\text { multiple kartilaginäre Exostosen; } \\
\mathrm{n}=84 \text { ) }\end{array}$ & 711 \\
\hline $\begin{array}{l}\text { Enchondrom (inklusive } \\
\text { Enchondromatose; } n=29 \text { ) }\end{array}$ & 640 \\
\hline Periostales/parossales Chondrom & 88 \\
\hline Chondroblastom & 104 \\
\hline Chondromyxoidfibrom & 39 \\
\hline \multicolumn{2}{|c|}{$\begin{array}{l}\text { Verteilung gut- und bösartiger Knorpeltumoren } \\
\text { im Register des Basler Knochentumor-Referenz- } \\
\text { zentrums (1972-2006) }\end{array}$} \\
\hline
\end{tabular}




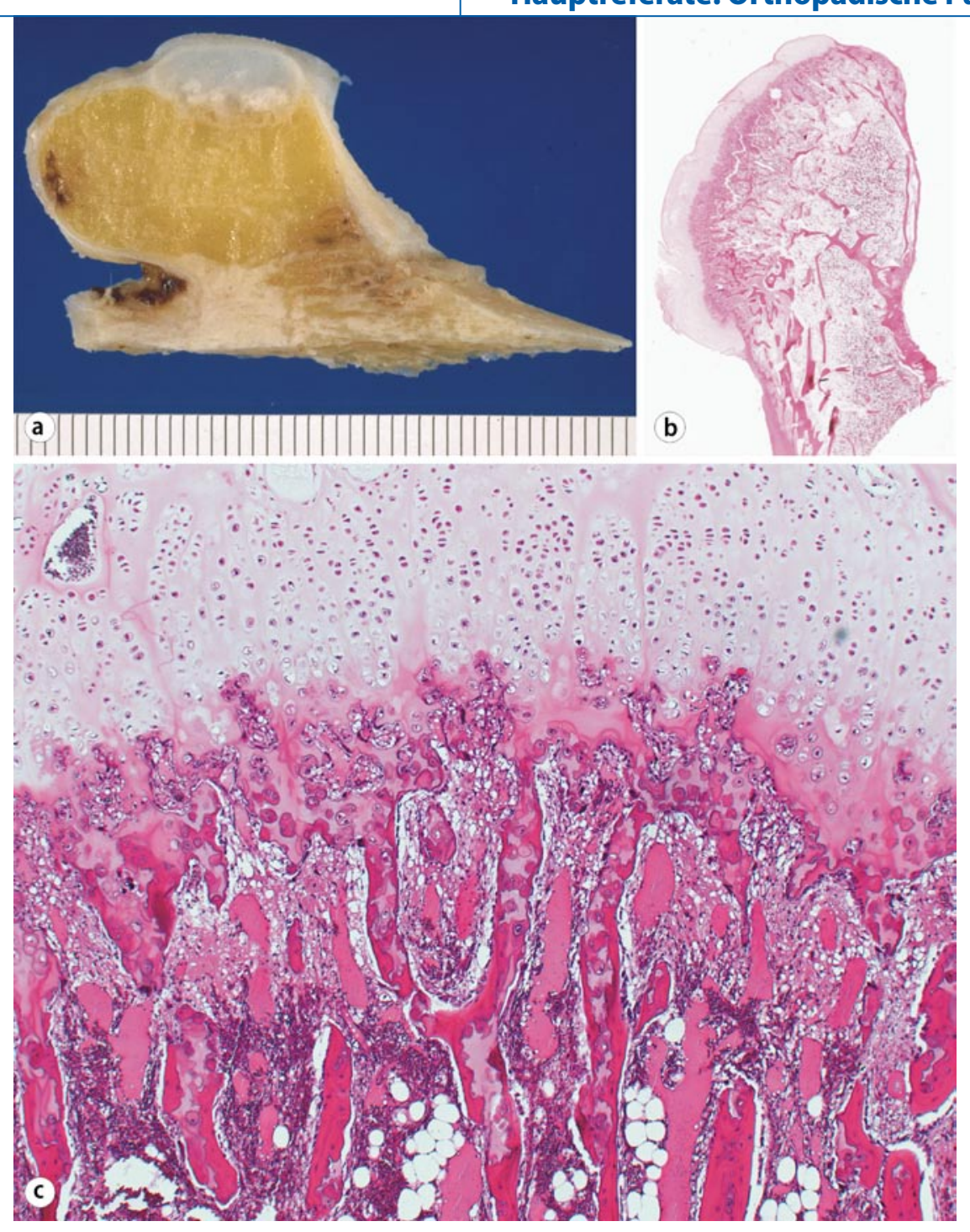

Abb. $1 \Delta$ Osteochondrom. a Gestieltes Osteochondrom (Resektionspräparat) mit partiell erhaltener Knorpelkappe. Die Kortikalis geht in den Stiel des Osteochondroms über. b Unterhalb der Knorpelkappe und in Kontinuität mit dem Markraum des betroffenen Knochens befindet sich Spongiosa mit Fettmark. Großschnitt (HE, Vergr:: 1:1). c Die Chondrozyten sind angedeutet säulenartig angeordnet und gehen in eine enchondrale Ossifikationszone über (HE, Vergr.: 1:50)

lenförmig angeordnete Chondrozyten gehen über eine enchondrale Ossifikationszone in spongiösen Knochen über, der von Fettmark und blutbildendem Mark umgeben wird (• Abb. 1). Die Breite der Knorpelkappe beträgt meist nur wenige Millimeter. Sollte sie $2 \mathrm{~cm}$ überschreiten, besteht besonders dann der Verdacht auf einen (sehr seltenen) Übergang in ein sekundäres „epiexostotisches“ Chondrosarkom, wenn die Läsion nach Abschluss der Pubertät gewachsen ist [7].

Multiple kartilaginäre Exostosen oder multiple Osteochondrome (MIM \#133700) sind autosomal-dominant vererbte, genetisch heterogene Erkrankungen, die durch
Alterationen in einem der drei EXT- Gene verursacht werden, in etwa $2 / 3$ der Fälle bei Männern vorkommen und eine Prävalenz von 1/50.00o aufweisen [5]. Während eine maligne Transformation in sporadisch vorkommenden Osteochondromen sehr selten ist, wird sie bei multiplen hereditären Osteochondromen oder Exostosen in bis zu $8 \%$ beobachtet $[5,20]$. In beiden Fällen ist sie durch das Auftreten einer myxoiden Matrix, den Verlust der säulenartigen Knorpelzellanordnung, zelluläre Atypien, Mitosen und die Infiltration des angrenzenden Weichgewebes charakterisiert.

\section{Chondrome}

Enchondrom, periostales Chondrom, Enchondromatose

Das Enchondrom ist ein hyalinknorplig differenzierter Tumor, der intramedullär oft in den kleinen Röhrenknochen besonders der Hände, aber auch in den langen Röhrenknochen (Femur, Tibia, Fibula) v. a. metadiaphysär vorwiegend bei Patienten im (3. bis) 4. Lebensjahrzehnt vorkommt [12]. Meist handelt es sich um Zufallsbefunde, gelegentlich treten auch pathologische Frakturen (Hände) auf. Schmerzen sind eher untypisch und sollten, besonders bei Lokalisation in den langen Röhrenknochen oder im Becken, an ein Chondrosarkom denken lassen. Da Enchondrome meist kürettiert werden, finden sich histologisch Fragmente hyalinknorpeligen Gewebes, das gelegentlich Verkalkungen aufweisen und verknöchern kann (• Abb. 2) [6]. Diese Knorpelossifikationen dürfen weder mit einer Osteodestruktion präexistenten lamellären Knochens noch mit einem Markraum ausfüllenden Wachstum verwechselt werden, bei dem Tumorknorpel ortsständige Spongiosabälkchen allseits umgibt und wie in die Markräume „ausgegossen“ wirkt. Für die Diagnose kommt dem Wachstumsmuster eine größere Bedeutung als dem Zellreichtum zu, wobei die Zellularität (Ausnahme: kleine Röhrenknochen!) eher niedrig ist und Hyperzellularität an ein Chondrosarkom denken lassen muss. Die Kerne sind pyknotisch-hyperchromatisch; Doppelkerne oder ein sog. offenes Chromatin (sichtbare kleine Nukleolen) kommen gelegentlich (in den kleinen Röhrenknochen häufiger) vor ( $\bullet$ Abb. 2d). Die Therapie besteht in einer Kürettage.

Die seltenen, meist bis $3 \mathrm{~cm}$ großen periostalen Chondrome entwickeln sich unterhalb des Periostes auf der Kortikalis vorwiegend der langen (Humerus), aber auch der kleinen Röhrenknochen und zeigen radiologisch eine charakteristische sklerosierte Eindellung der verbreiterten Kompakta. Die extraperiostalen Weichteile sind nicht befallen (DD: periostales Chondrosarkom); der angrenzende Markraum enthält allenfalls oberflächlich gelegene Noduli ohne Markraumpermeation 

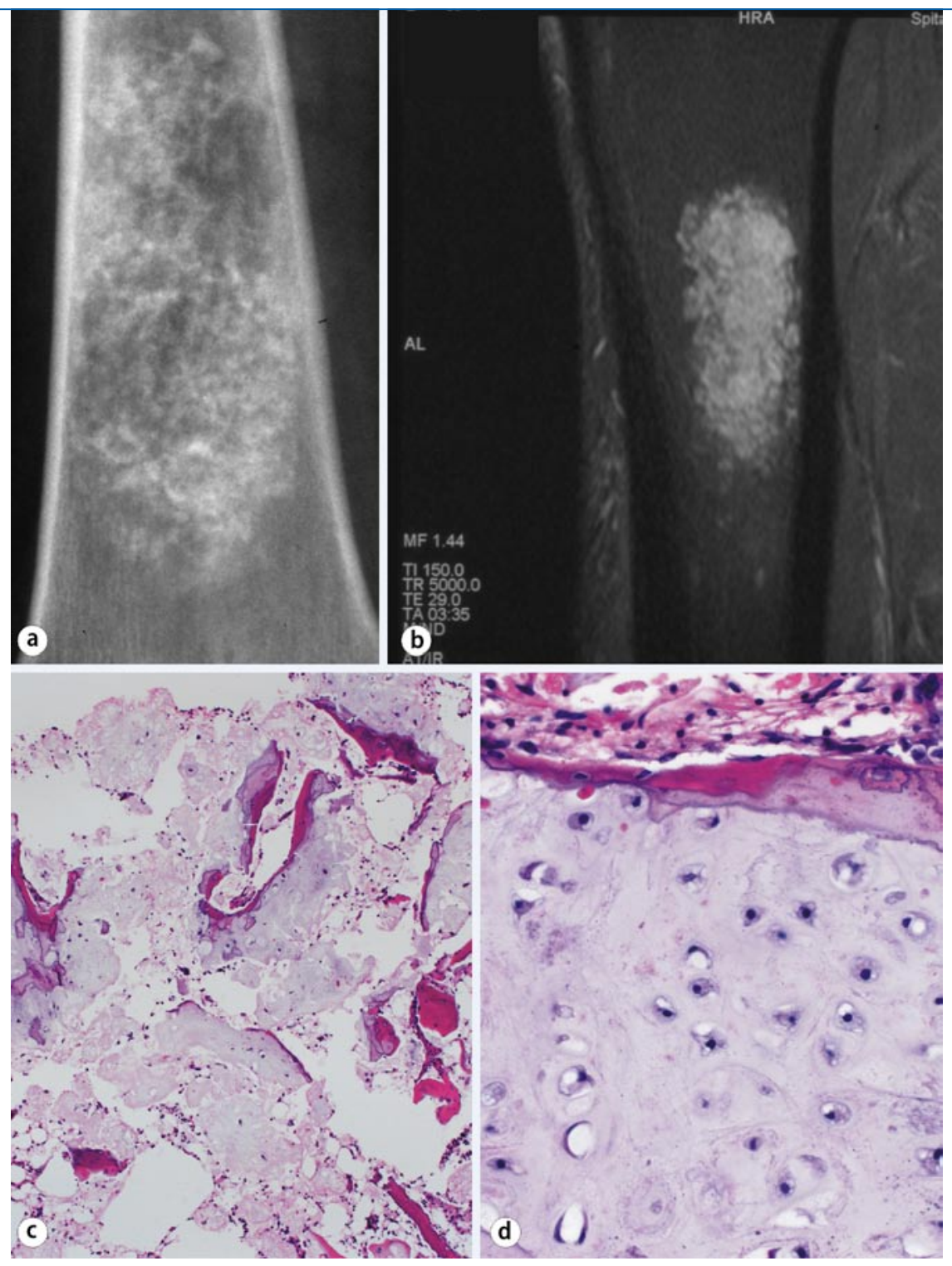

Abb. $2 \Delta$ Enchondrom. a Konventionelles Röntgenbild, a.-p.: Irreguläre längliche Verkalkungszone im distalen Drittel des rechten Femurs mit angedeutet bogigen und kreisförmigen Kalzifikationen. $\mathbf{b}$ Im spongiosafreien Knochen (proximale Femurdiaphyse) lässt sich ein nicht verkalkendes Enchondrom nur durch sein Signalverhalten im MRT darstellen. Der hohe Flüssigkeitsgehalt des Knorpelgewebes führt zu sehr hohen (hellen) Signalintensitäten bei speziellen Techniken (hier T2-Gewichtung mit Fettsättigung). c Ineinander geschobene Kürettagefragmente eines Enchondroms. Die periphere Ossifikation der partiell erhaltenen Knorpelläppchen kann eine Markrauminfiltration vortäuschen (HE, Vergr. 1:25). d Peripherie Ossifikation eines Enchondromnodulus, korrespondierend zu den Verkalkungen in a. Die Zellkerne sind rund und zeigen ein dichtes Chromatin (HE, Vergr. 1:400)

(- Abb. 3a, b). Die Zellularität ist etwas gesteigert, Kernpleomorphie und Doppelkernigkeit sind leicht erhöht. Exzision oder Kürettage sind die bevorzugten Therapien $[7,12]$.

Unter einer Enchondromatose (MIM\# 1660oo) versteht man eine sehr seltene, meist nichthereditäre Störung der Knorpelentwicklung, die zu zahlreichen, oft einseitig lokalisierten Enchondromen und periostalen Chondromen führt (M. Ollier) und in Kombination mit extraskelettalen Häm- angiomen (Maffucci-Syndrom) auftreten kann. Daneben kommen noch einzelne andere Formen vor $[12,22]$. Die Erkrankung beginnt bereits in der Kindheit und kann zu ausgeprägten Skelettdeformierungen führen. Die sich meist metaphysär entwickelnden Tumoren sind hyperzellulär, die Zellkerne vergrößert und eher länglich als rund, Doppelkerne sind zahlreich. Das Risiko einer Sarkomentwicklung liegt bei 15-30\%, wobei das Auftreten einer myxoiden Matrix und eine Infiltration der Mark-
Pathologe 2008 · [Suppl 2] 29:223-231

DOI 10.1007/s00292-008-1025-z

c) Springer Medizin Verlag 2008

\section{G. Jundt $\cdot$ D. Baumhoer Chondrogene Tumoren des Skeletts}

\section{Zusammenfassung}

Obwohl das Spektrum gutartiger und bösartiger Knorpeltumoren sehr breit ist, lässt sich unter Berücksichtigung von Alter, Klinik, Lokalisation und Topik im Knochen sowie des Röntgenbefundes in der Regel auch an kleinen Biopsien eine sichere Diagnose stellen. Dies gilt allerdings nur mit Einschränkungen für die Unterscheidung zwischen Enchondromen und Chondrosarkomen Grad 1 und setzt insgesamt eine langjährige Erfahrung in der interdisziplinären Knochentumordiagnostik voraus. In Einzelfällen können immunhistochemische Ergebnisse differenzialdiagnostisch weiterhelfen, wenn sie im Kontext der übrigen Befunde interpretiert werden. Wegen der therapeutischen und prognostischen Konsequenzen ist die Zusammenarbeit mit einem Referenzzentrum ratsam.

\section{Schlüsselwörter}

Knorpeltumoren · Gutartig · Bösartig ·

Histologie $\cdot$ Referenzzentren

\section{Cartilage tumors of the skeleton}

\section{Abstract}

Although the spectrum of benign and malignant cartilaginous bone tumors is extremely wide, a distinct diagnosis, even from small biopsy specimens, is almost always possible if radiological findings, age, clinical data, and localization within the skeleton as well as within the bone are considered. With limitations, this also holds true for distinguishing enchondromas from low-grade chondrosarcomas; however, extensive experience in multidisciplinary bone tumor diagnosis is required. In single cases, immunohistochemical findings may be helpful in the differential diagnosis if they are integrated into the context of all other findings. Because of treatment-related or prognosis-related consequences, collaboration with a reference center is recommended.

\section{Keywords}

Bone tumors - Cartilage - Benign - Malignant . Histology $\cdot$ Reference center 


\section{Hauptreferate: Orthopädische Pathologie}
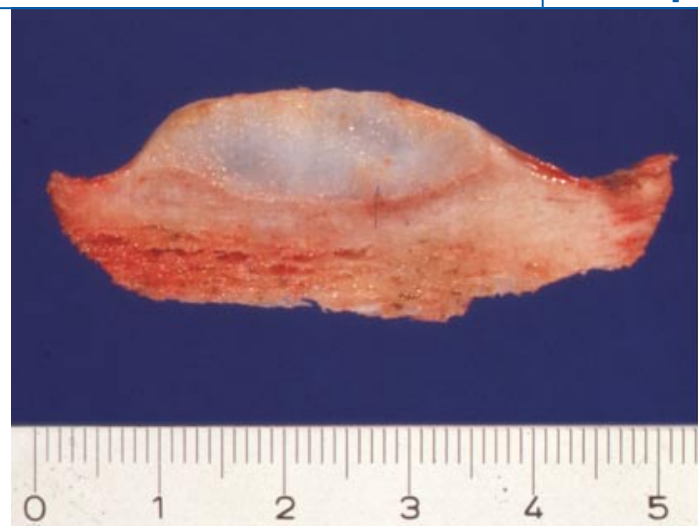

a
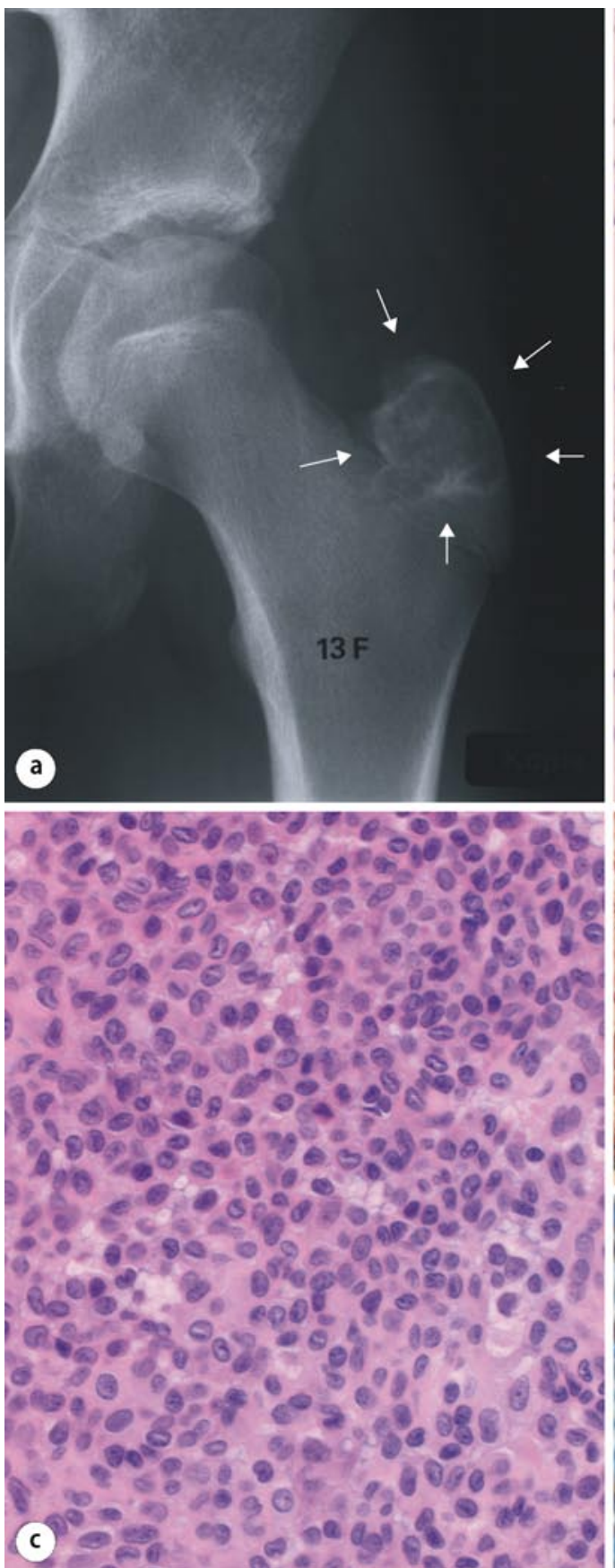
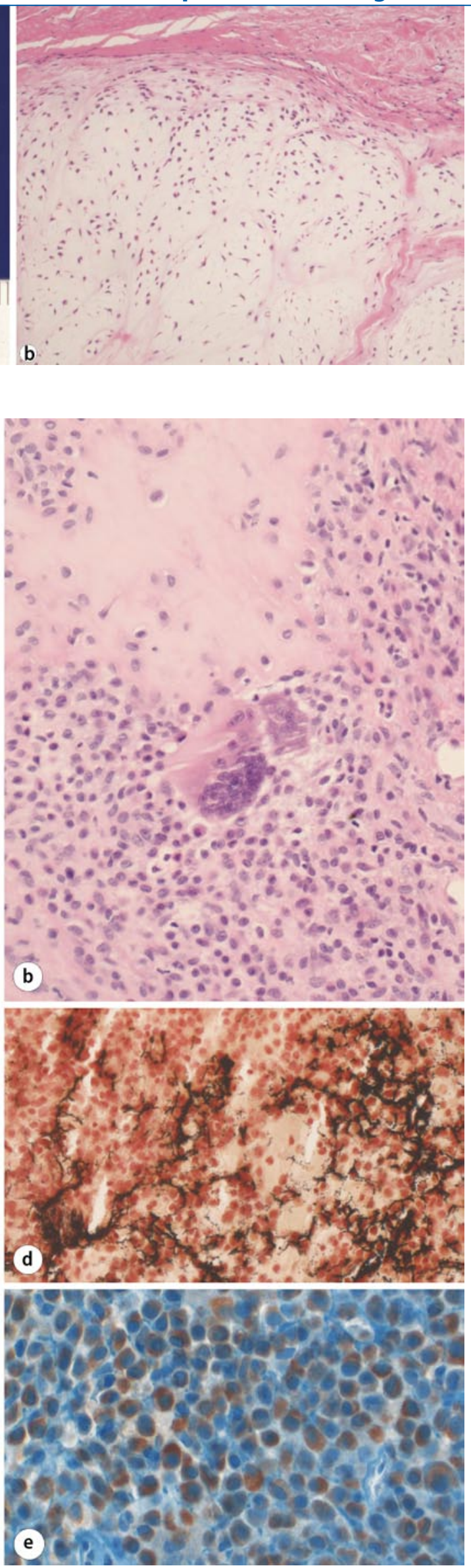

Abb. $3<$ Periostales Chondrom. a Resektionspräparat eines periostalen Chondroms. Klare Begrenzung des Tumors zur Tiefe (Kortikalisoberfläche). Die Kortikalis selbst ist bereits makroskopisch stark verbreitert. b Zelldichter hyaliner Knorpel mit relativ gleichförmigen, etwas entrundeten Kernen ohne Durchbruch des Perichondriums (HE, Vergr. 1:100)
Abb. $4<$ Chondroblastom. a Röntgenübersicht, a.-p.: In der Trochanterapophyse gelegene Osteolyse (Pfeile), die den Knochen leicht auftreibt und einen inkompletten Sklerosesaum besitzt. b Neben relativ isomorphen mononukleären Zellen finden sich Riesenzellen vom Osteoklastentyp. In der oberen Bildhälfte sieht man die für das Chondroblastom typische chondroosteoide Matrix (HE, Vergr. 1:200). c Die Tumorzellen besitzen ein mittelbreites eosinophiles Zytoplasma, distinkte Zellgrenzen und oft eingekerbte Kerne (HE, Vergr. 1:630). d In der Kossa-Färbung sieht man die typische maschendrahtartige perizelluläre Mineralisierung (Kossa, Vergr. 1:200). e Positive Reaktion der Chondroblastomzellen mit einem Panzytokeratinantikörper (CK22, Vergr. 1:630) 
räume mit Destruktion der Spongiosa diagnostisch wegweisend sind.

\section{Chondroblastom}

Chondroblastome sind sehr seltene gutartige, fast ausschließlich epi(meta)physär auftretende Tumoren, die vorwiegend in den langen Röhrenknochen junger männlicher Patienten (im 2. Lebensjahrzehnt) vorkommen und radiologisch durch eine scharf begrenzte Osteolyse oft mit Sklerosesaum gekennzeichnet sind ( $\bullet$ Abb. 4a). Sie machen sich durch eine leichte, oft langjährig bestehende Schmerzsymptomatik bemerkbar [11].

Histologisch sieht man rundlich-ovale Zellen mit sehr gut erkennbaren Grenzen. Das Zytoplasma ist meist nur mäßig verbreitert und blass-eosinophil. Die runden bis längs ovalen Kerne zeigen oft kaffeebohnenartige Einfaltungen. Daneben finden sich osteoklastäre Riesenzellen, deren Kernzahl deutlich niedriger ist als im ebenfalls epimetaphysär vorkommenden Riesenzelltumor. Die Zellen sind in eine Matrix eingebettet, die nur selten und abschnittsweise hyalinem Knorpel entspricht und meist einen eher blasseosinophilen, „chondroosteoiden“ Charakter hat. Typisch ist eine feine, maschendrahtähnliche perizelluläre Mineralisation, die in der Kossa-Färbung (unentkalkte Präparate!) gut zum Ausdruck kommt (- Abb. 4b, c, d). Mit der Zeit können Ossifikationen auftreten. Gelegentlich sind Areale einer aneurysmatischen Knochenzyste vorhanden [24]. Immunhistochemisch zeigen die Tumorzellen neben S100 und Vimentin eine ausgeprägte Zytokeratinexpression ( $\bullet$ Abb. 4e) [11]. Trotz dieser und anderer morphologischer sowie immunhistochemischer Besonderheiten (keine Typ-II-Kollagenproduktion) werden Chondroblastome zu den knorpelbildenden Tumoren gezählt [1, 21]. Therapeutisch ist in der Regel eine Kürettage ausreichend, Rezidive sind aber möglich. Wie bei Riesenzelltumoren sind Lungenmetastasen auch bei Chondroblastomen in Einzelfällen beschrieben worden [24]. Die Differenzialdiagnose muss v. a. gegen den Riesenzelltumor (kein Sklerosesaum, keine Kerneinkerbungen, undeutliche Grenzen der mononukleären Zellen, kein Chondroosteoid, Zytokera-
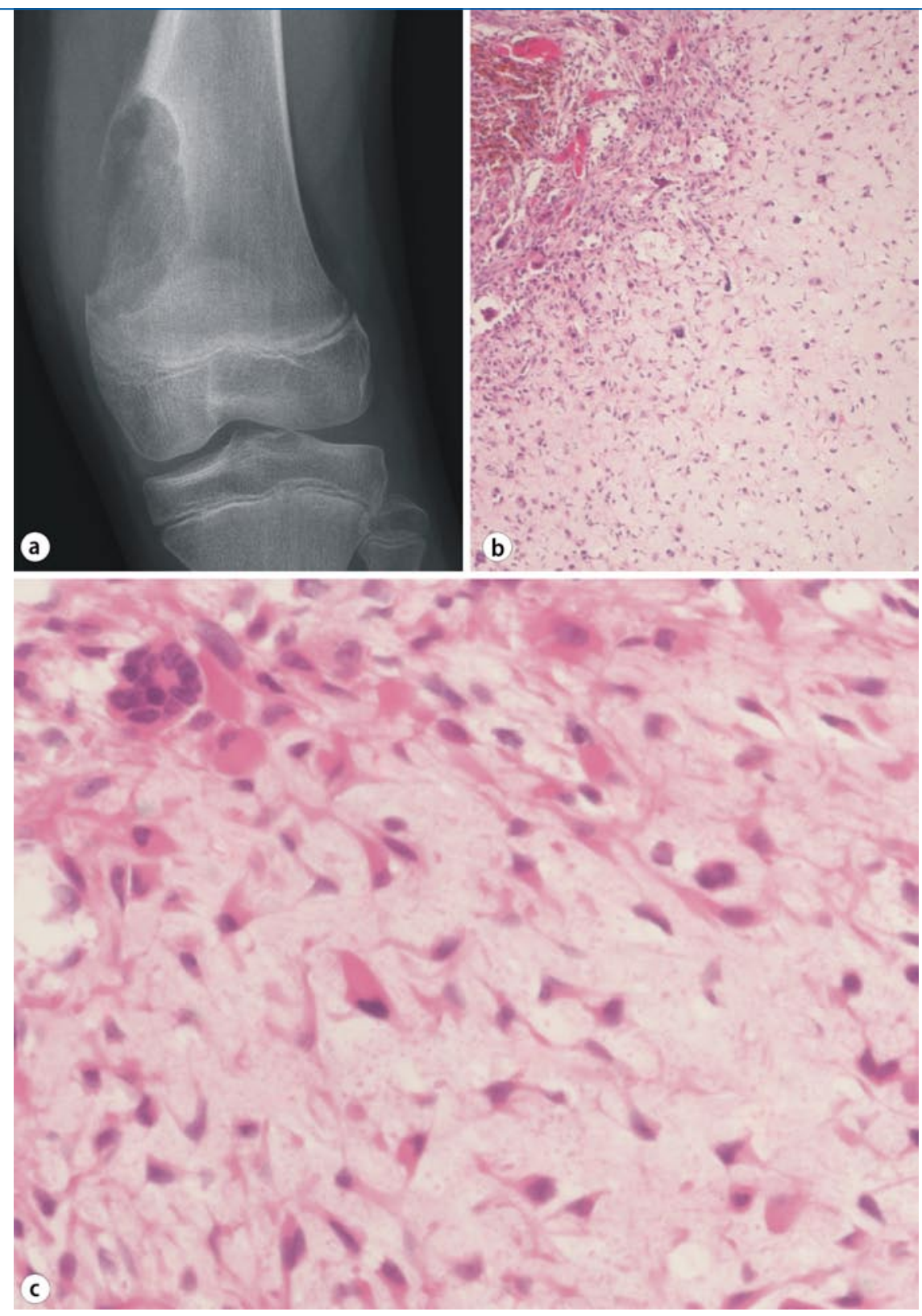

Abb. $5 \Delta$ Chondromyxoidfibrom. a Röntgenübersicht, a.-p.: exzentrische Osteolyse der distalen Femurmetaphyse mit Kortikalisdurchbruch. b Das läppchenförmig angeordnete Tumorgewebe zeigt ein myxoides Stroma mit Kondensation der Tumorzellen in der Läppchenperipherie. Hier sieht man außerdem Blutgefäße, Rundzellinfiltrate und Riesenzellen (HE, Vergr. 1:100). c Die teils dreieckförmigen Zellen besitzen zum Teil große, chromatindichte, irregulär konfigurierte Kerne (HE, Vergr. 1:400)

tin negativ), das Osteosarkom (Kernpolymorphie, osteodestruktives Wachstum) und das Chondromyxoidfibrom (metaphysär gelegen, lobulärerer Aufbau mit Zunahme der Zellzahl in der Läppchenperipherie, Zytokeratin negativ) erfolgen.

\section{Chondromyxoidfibrom}

Das Chondromyxoidfibrom ist ein vorwiegend in den Metaphysen der Röhrenknochen, besonders der proximalen Tibia- metaphyse, aber auch im Becken und den Rippen auftretender, exzentrisch gelegener osteolytischer Tumor, der radiologisch nur selten knorpeltypische bogenoder ringförmige Matrixkalzifikationen aufweist (- Abb. 5a). Er kommt etwas häufiger bei Männern als bei Frauen vor, meist im 2.-3. Lebensjahrzehnt und verursacht oft leichte Schmerzen, gelegentlich auch Schwellungen [19].

Histologisch fällt bereits in der Übersicht der lobuläre Aufbau der Läsion auf. 

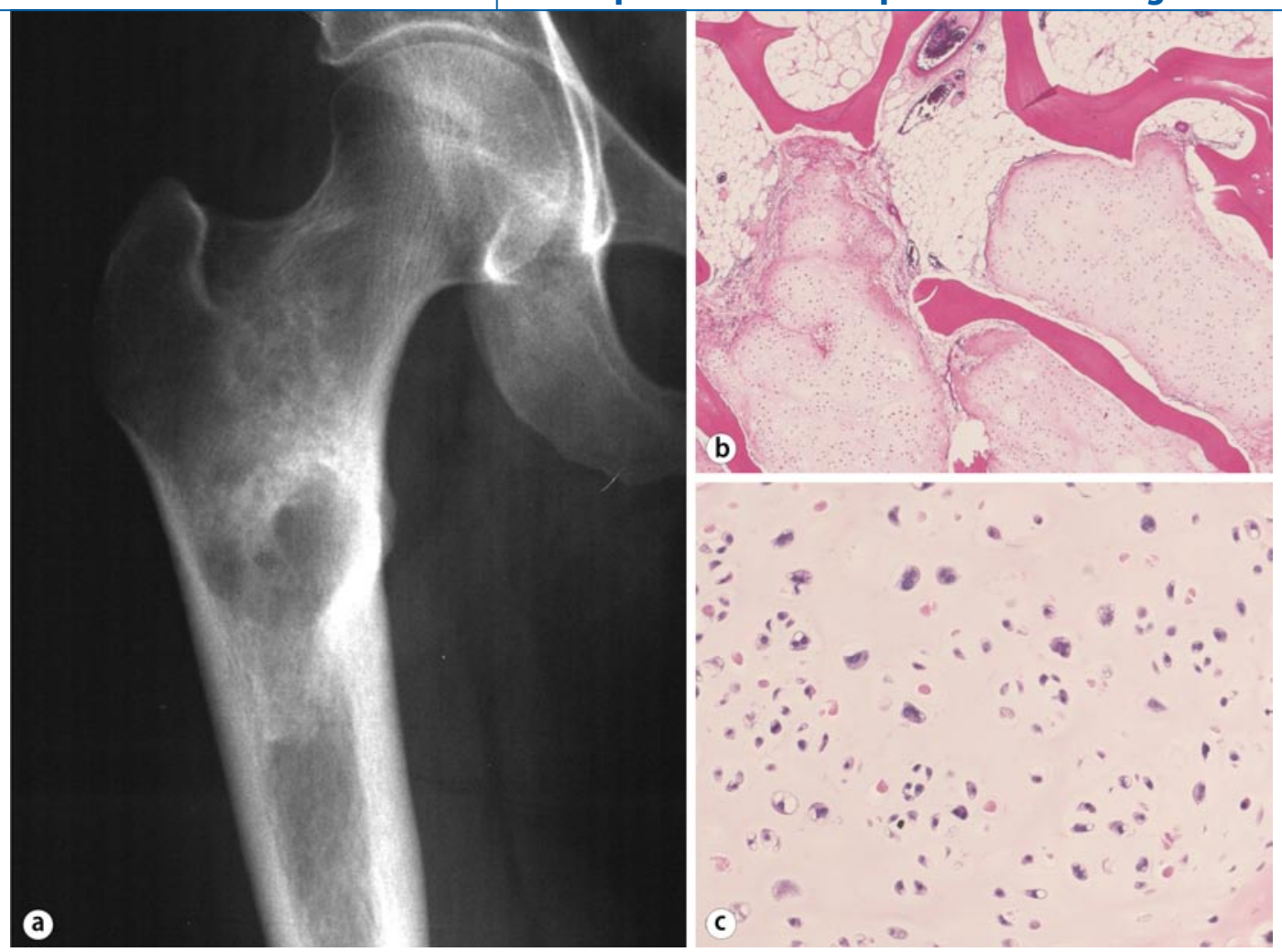

Abb. $6<$ Chondrosarkom. a Röntgenübersicht, a.-p.: Die proximale Femurdiaphyse zeigt eine Osteolyse, die zu einer Ausbuchtung der medialen Kortikalis geführt hat. Im Trochantermassiv erkennt man ringförmige Verkalkungsmuster als Hinweis auf einen chondrogenen Tumor. b Das Geschwulstgewebe umfließt die Spongiosabälkchen allseits und füllt die Markräume aus (HE, Vergr. 1:50). c Die Tumorzellen zeigen zum Teil deutlich vergrößerte Kerne, daneben sieht man Doppelkerne sowie Zellen mit hellem Kernchromatin (sog. "offenes Chromatin") und erkennbaren Nukleolen (HE, Vergr. 1:200)

Spindelige bis sternförmige Zellen sind in eine fibromyxoide Matrix eingebettet, wobei das gelegentlich verbreiterte eosinophile Zytoplasma gut erkennbar ist. Hyaliner Knorpel kommt nur in maximal 20\% der Fälle vor. Die zentral zellarmen Lobuli werden zur Peripherie hin zelldichter, sodass die Läppchen wie durch Septen getrennt erscheinen. In diesen zellreichen Arealen sind immer wieder osteoklastäre Riesenzellen zu finden (• Abb. 5b, c). Außerdem kommen Zellen mit chromatindichten, vergrößerten („pseudomalignen“) Kernen vor, Mitosen fehlen jedoch [26]. Differenzialdiagnostisch müssen Chondrosarkome (aggressivere Radiologie, stärkergradige Zellatypien, homogene Verteilung der Tumorzellen in den Lobuli), gelegentlich auch Chondroblastome (epiphysär, Kerneinbuchtungen, Matrixverkalkungen) abgegrenzt werden. Die Therapie besteht in einer Kürettage. Wegen der hohen Rezidivrate von etwa $20 \%$ wird oft auch eine lokale Exzisison vorgenommen.

\section{Chondrosarkome}

Nach dem Osteosarkom ist das Chondrosarkom der zweithäufigste maligne Knochentumor, das überwiegend im 5.-7. Lebensjahrzehnt vorwiegend bei Männern auftritt, aber auch im Jugendalter beobachtet werden kann. Chondrosarkome bei Kindern sind Raritäten. Etwa 80\% der malignen knorpelbildenden Tumoren sind klassische oder konventionelle Chondrosarkome, die vorwiegend in den langen Röhrenknochen, im Becken und in den Knochen des Thorax vorkommen [3].

In der Regel machen sie durch meist schon Monate bis Jahre lang bestehende Schmerzen und/oder Schwellungen auf sich aufmerksam. In den Röhrenknochen treten Chondrosarkome metadiaphysär-intramedullär auf und führen zu einer Auftreibung des Knochens mit Arrosionen und Aufblätterungen der Kortikalis, die im weiteren Verlauf durchbrochen wird (- Abb. 6a). Dem Röntgenbefund kommt ebenso wie der Anamnese (Schmerzen) bei der Diagnose eine erhebliche Bedeutung zu, da gerade niedrigmaligne Chondrosarkome sehr heterogen aufgebaut sein können und oft ausgedehnte Areale aufweisen, die von einem Enchondrom histologisch nicht zu unterscheiden sind [9].

Beweisend für den malignen Charakter sind ein Markraum ausfüllendes Wachstum und eine begleitende Osteodestruktion [6]. Daneben finden sich doppelkernige Zellen und zahlreiche Zellen mit zum Teil „offenen“ Chromatin (d. h. sicht- baren Nukleolen), diese Befunde können jedoch auch bei Enchondromen vorkommen. Die Tumorzellen werden von einer bläulichen hyalinknorpeligen Matrix umgeben und liegen in Lakunen, wobei das Tumorgewebe lobulär angeordnet ist (- Abb. 6b, c). Mit steigendem Malignitätsgrad zeigt die Matrix einen myxoiden Charakter; Zellulariät und Atypiegrad nehmen zu und spindelige Tumorzellen treten auf. Der lobuläre Tumoraufbau geht mehr und mehr verloren [24]. Basierend auf diesen Befunden können Chondrosarkome in 3 Malignitätsgrade eingeteilt werden [25]. Dies hat prognostische Bedeutung, da Grad-1-Chondrosarkome praktisch nur zu Rezidiven neigen, während Grad-2- (bis 20\%) und Grad-3-Sarkome (bis 50\%) zunehmend Metastasen entwickeln [8].

Differenzialdiagnostisch müssen Enchondrome (nichtaggressiver Röntgenbefund, weder Osteodestruktion noch Markraum ausfüllendes Wachstum) und chondroblastische Osteosarkome (Jugendliche, radiologisch flächige Verkalkungsmuster, Tumorosteoidbildung) abgegrenzt werden.

Die Therapie besteht bei Grad-1-Chondrosarkomen in der Exzision, bei Grad-2- 
und -3-Tumoren in einer weiten Resektion.

Extrem selten können sich Chondrosarkome auch auf der Knochenoberfläche (meist distale Femurmetaphyse) als periostale Chondrosarkome entwickeln. Diese Tumoren sind lokal aggressiv, größer als periostale Chondrome $(>5 \mathrm{~cm})$ und in filtrieren im Gegensatz zu letzteren das angrenzende Weichgewebe. Metastasen können vorkommen [18].

\section{Dedifferenziertes Chondrosarkom}

Dedifferenzierte Chondrosarkome sind seltene, hochmalige Knochentumoren, die etwa 10\% aller Chondrosarkome ausmachen und hauptsächlich im 6. und 7. Lebensjahrzehnt auftreten, bei Männern etwas häufiger als bei Frauen.

Meist sind sie in den langen Röhrenknochen (Femur, Humerus) oder im Becken lokalisiert. Schmerzen, Schwellungen oder pathologische Frakturen sind die führenden Symptome.

Der typische radiologische Befund zeigt eine intraossär gelegene Sklerosezone, die einen Knorpeltumor mit ringförmigen Verkalkung repräsentiert, der mit einer aggressiveren, oft expansiven und osteodestruktiven lytischen Läsion kombiniert ist [15].

Dieser Befund wird histologisch durch den Übergang eines Enchondroms oder Chondrosarkoms Grad 1 in ein High-gradeSarkom hervorgerufen, wobei charakteristischerweise beide Tumorkomponenten nebeneinander bestehen und nicht miteinander vermischt sind (• Abb. 7a, b).

Die High-grade-Komponente kann aus einem konventionellen Osteosarkom, einem pleomorphen (MFH-like-)Sarkom, einem Fibrosarkom oder - sehr selten - einem Rhabdomyosarkom bestehen, gelegentlich aber auch einen epitheloiden Charakter haben, der an Metastasen denken lässt [24]. Für die Diagnosestellung ist die Korrelation der Histologie mit dem Röntgenbefund entscheidend, da nicht selten nur eine Komponente biopsiert wird [9]. Die Therapie wird heute im Rahmen von Studien durchgeführt (EuroBOSS: EUROpean Bone Over 40 Sarcoma Study: alle High-grade-Sarkome im Knochen bei Patienten über 40 Jahren; Studienleitung in Stuttgart, Prof. S. Bielack, Ol-

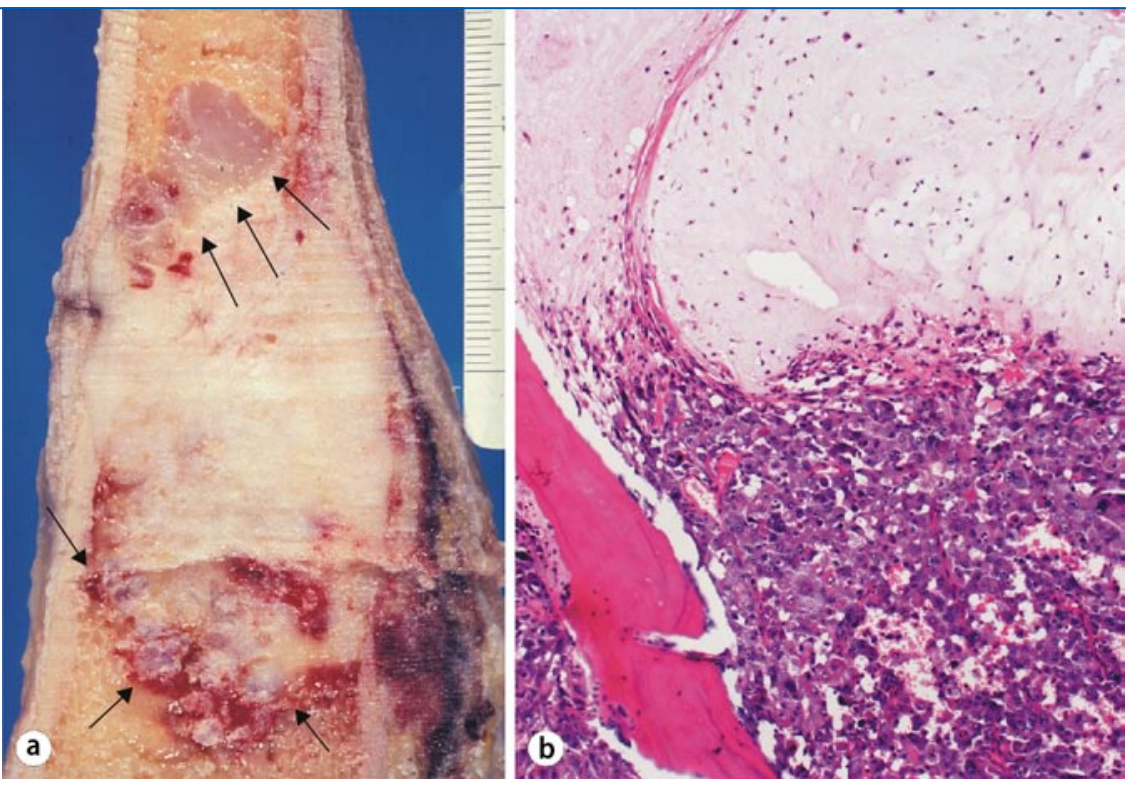

Abb. 7 A Dedifferenziertes Chondrosarkom. a Das Resektionspräparat zeigt kompakte, knorpelig differenzierte Tumoranteile (Pfeile), die an ein fischfleischartiges Geschwulstgewebe grenzen. b Hochdifferenziertes Chondrosarkom Grad 1 und wenig differenziertes MFH-artiges Sarkom grenzen typischerweise unmittelbar aneinander, ohne sich zu durchmischen (HE, Vergr. 1:100)
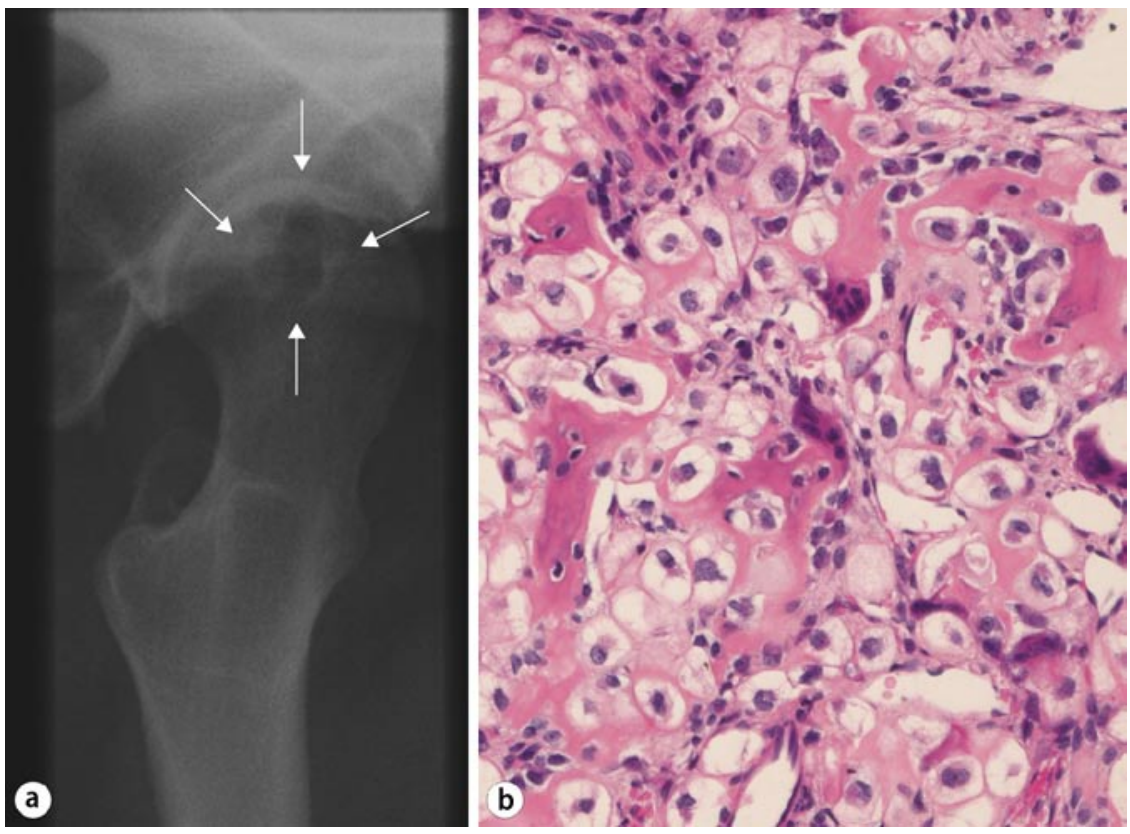

Abb. $8 \Delta$ Klarzellchondrosarkom. a Röntgenübersicht, Lauenstein: Typisch gelegene epiphysäre Osteolyse (Pfeile) unterhalb der Gelenkfläche des Femurkopfes. b Großleibige, helle Tumorzellen mit großen, meist zentralen Kernen und gut erkennbaren Zellgrenzen, daneben einzelne Osteoklasten und neugebildetes (tumorinduziertes) Osteoid (HE, Vergr. 1:200)

gaspital) und besteht in einer Kombination von weiter Resektion und Chemotherapie. Die Prognose wird von der Highgrade Komponente bestimmt. Nach eigenen Erfahrungen an 45 Fällen beträgt die 5-Jahres-Überlebensrate bis zu 35-40\%.

\section{Klarzellchondrosarkom}

Das sehr seltene (unter $2 \%$ aller Chondrosarkome), niedrigmaligne Klarzellchondrosarkom lässt sich durch seine bevorzugte Lokalisation (Epiphyse der langen Röhrenknochen, v. a. proximales Femur), sein Auftreten um das 40. Lebensjahr und die Bevorzugung des männ- 


\section{Hauptreferate: Orthopädische Pathologie}
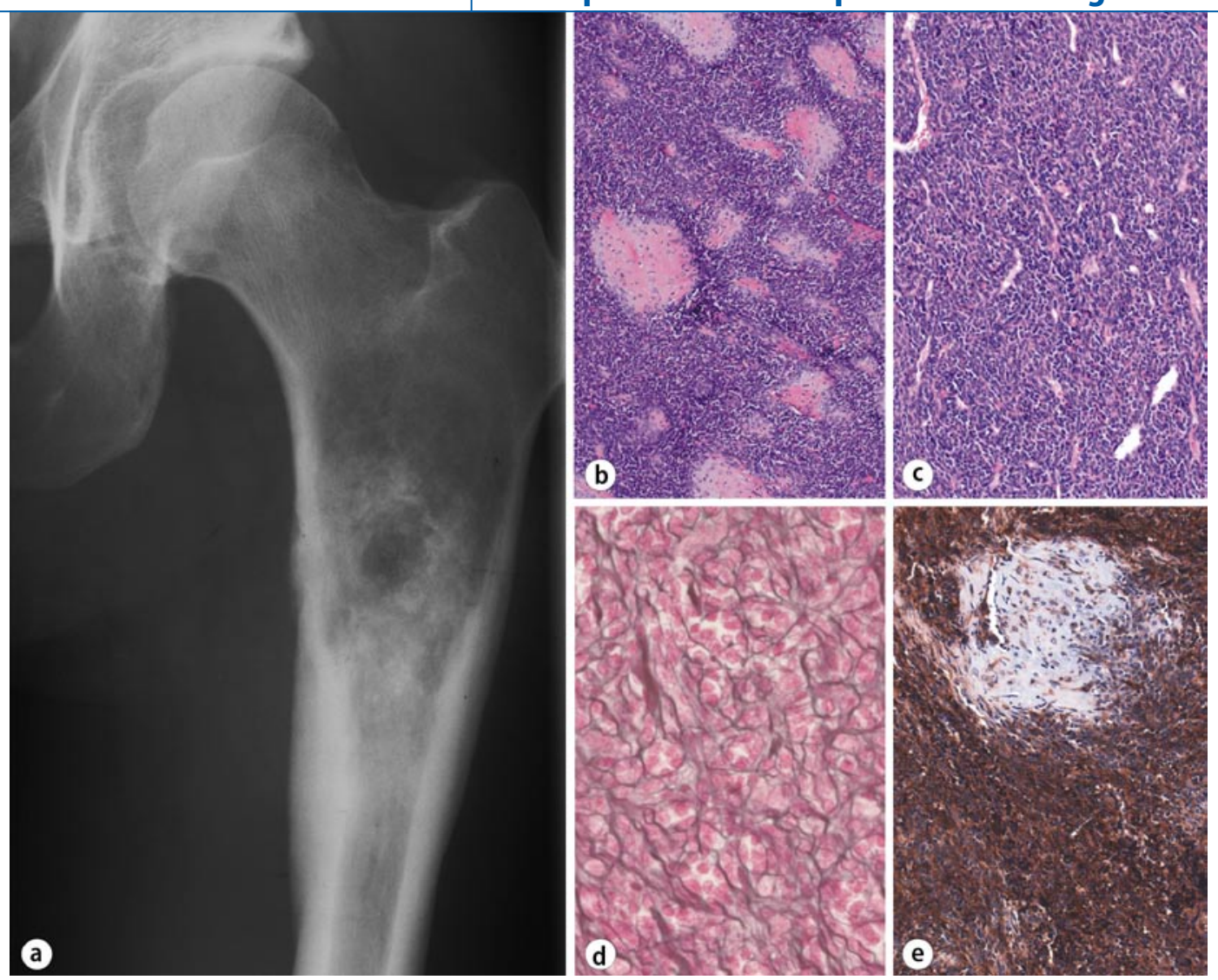

Abb. $9<$ Mesenchymales Chondrosarkom. a Röntgenübersicht, a.-p: Osteolyse der proximalen Femurdiaphyse umgeben von teils ringförmigen Verkalkungen. Die Kortikalis ist arrodiert. An der Außenseite ist eine feine lamelläre Periostreaktion erkennbar. b Nebeneinander von Knorpelinseln und klein-, rund- und blauzelligen Tumorarealen (HE, Vergr. 1:50). c Abschnittsweise sind die Tumorzellen hämangioperizytomartig angeordnet (HE, Vergr. 1:100). d Kleine Gruppen und einzelne Tumorzellen werden von Retikulinfasern umsponnen (Novotny, Vergr. 1:630). e Die undifferenzierten Tumorzellen reagieren positiv mit $\mathrm{CD} 99$ (Vergr. 1:200)

lichen Geschlechts bereits klinisch vom konventionellen Chondrosarkom abgrenzen (• Abb. 8a). Es macht sich durch längerdauernde, manchmal jahrelange leichte Schmerzen oder eine pathologische Fraktur bemerkbar [14].

Histologisch ist der Tumor durch große, gut begrenzte Zellen mit breitem, hellem bis blass-eosinophilem Zytoplasma und deutlich erkennbaren mittelständigen, rundlichen Kernen gekennzeichnet, die einen gut sichtbaren $\mathrm{Nu}$ kleolus enthalten. Mitosen sind selten. Das Geschwulstgewebe ist lobuliert, zwischen den Tumorzellen finden sich osteoklastäre Riesenzellen und neugebildetete Geflechtknochenbälkchen, die Anlass zu Verwechslungen mit einem Osteosarkom geben können ( $\bullet$ Abb. 8b). Hyalinknorpelig differenzierte Areale kommen in unterschiedlichem (meist geringen) Ausmaß vor [4]. Differenzialdiagnostisch muss neben dem Osteosarkom (metaphysär, jüngere Patienten, ausgeprägtere Zell- und Kernplymorphie, Tumorosteoid) auch an ein Chondroblastom (Sklerosesaum im Röntgenbild, jüngere Patienten, Kerneinkerbungen, Zytokeratinpositivität) oder Metastasen eines hellzel- ligen Nierenzellkarzinoms (keine Riesenzellen oder Knochenbälkchen, RCC-positiv) gedacht werden. Die Therapie besteht in einer weiten Resektion, andernfalls treten Rezidive auf.

\section{Mesenchymales Chondrosarkom}

Mesenchymale Chondrosarkome sind hochmaligne, oft auch extraskelettal auftretende Tumoren, die meist im 2. bis 3. Lebensjahrzehnt vorkommen und häufig die Kiefer, die Rippen oder das Becken betreffen. Alle Knochen können jedoch befallen sein. Radiologisch präsentieren sich die Tumoren als destruktive osteolytische Läsionen( $\bullet$ Abb. 9a) [16]. Charakteristischerweise sind die Tumoren biphasisch aufgebaut und bestehen aus kleinen Inseln allenfalls gering atypischen hyalinen Knorpels, der von undifferenzierten rundlichen Zellen umgeben wird (• Abb. 9b). Teilweise zeigen die undifferenzierten Zellen eine hämangioperizytomähnliche Anordnung, teilweise erinnern sie an ein EwingSarkom (• Abb. 8b, d) [17]. Im Gegensatz zum Ewing-Sarkom lassen sich nach eigenen Erfahrungen immer Retikulinfa- sern nachweisen, die einzelne oder kleine Gruppen von Tumorzellen umgeben (- Abb. 8e). Die undifferenzierten Zellen sind CD99-positiv, zeigen jedoch keine Signalaufsplitterung in FISH-Breakapart-Untersuchungen mit der EWSR1Probe bzw. keine $\mathrm{t}(11 ; 22)$-Translokation (- Abb. 8c). Die Behandlung besteht in einer weiten Resektion, Radiotherapie und gegebenenfalls Chemotherapie.

\section{Korrespondenzadresse}

\section{Prof. Dr. G. Jundt}

Knochentumor-Referenzzentrum, Institut für Pathologie des Universitätsspitals Basel Schönbeinstr. 40, 4031 Basel, Schweiz gernot.jundt@unibas.ch

Interessenkonflikt. Der korrespondierende Autor gibt an, dass kein Interessenkonflikt besteht.

\section{Literatur}

1. Aigner T, Loos S, Inwards C et al. (1999) Chondroblastoma is an osteoid-forming, but not cartilageforming neoplasm. J Pathol 189: 463-469

2. Ashwood N, Witt JD, Hallam PJ et al. (2003) Analysis of the referral pattern to a supraregional bone and soft tissue tumour service. Ann R Coll Surg Engl 85: 272-276 
3. Bertoni F, Bacchini P, Hogendoorn PC (2002) Chondrosarcoma. In: Fletcher CDM, Unni KK, Mertens F (eds) WHO: Pathology and genetics of tumours of the soft tissue and bone. IARC Press, Lyon, pp 247251

4. Bjornsson J, Unni KK, Dahlin DC et al. (1984) Clear cell chondrosarcoma of bone. Observations in 47 cases. Am J Surg Pathol 8: 223-230

5. Bovée J, Hogendoorn P (2002) Multiple osteochondromas. In: Fletcher CDM, Unni KK, Mertens F (eds) WHO: Pathology and genetics of tumours of the soft tissue and bone. IARC Press, Lyon, pp 360-362

6. Brien EW, Mirra JM, Kerr R (1997) Benign and malignant cartilage tumors of bone and joint: their anatomic and theoretical basis with an emphasis on radiology, pathology and clinical biology. I. The intramedullary cartilage tumors. Skeletal Radiol 26: 325-353

7. Brien EW, Mirra JM, Luck JV jr (1999) Benign and malignant cartilage tumors of bone and joint: their anatomic and theoretical basis with an emphasis on radiology, pathology and clinical biology. II. Juxtacortical cartilage tumors. Skeletal Radiol 28: 1-20

8. Campanacci M (1999) Bone and soft tissue tumors. Springer, Berlin Heidelberg New York Tokio

9. Freyschmidt J, Ostertag H, Jundt G (1998) Knochentumoren. Klinik. Radiologie. Pathologie. Springer, Berlin Heidelberg New York Tokio

10. Khurana J, Abdul-Karim F, Bovée J (2002) Osteochondroma. In: Fletcher CDM, Unni KK, Mertens F (eds) WHO: Pathology and genetics of tumours of the soft tissue and bone. IARC Press, Lyon, pp 234236

11. Kilpatrick S, Parisien M, Bridge J (2002) Chondroblastoma. In: Fletcher CDM, Unni KK, Mertens F (eds) WHO: Pathology and genetics of tumours of the soft tissue and bone. IARC Press, Lyon, pp 241242

12. Lucas D, Bridge JA (2002) Chondromas: enchondroma, periosteal chondroma, and enchondromatosis. In: Fletcher CDM, Unni KK, Mertens F (eds) WHO: Pathology and genetics of tumours of the soft tissue and bone. IARC Press, Lyon, pp 237-240

13. Mankin HJ, Mankin CJ, Simon MA (1996) The hazards of the Biopsy, Revisited. J Bone Joint Surg Am 78-A: 656-663

14. McCarthy EF, Freemont A, Hogendoorn PWC (2002) Clear cell chondrosarcoma. In: Fletcher CDM, Unni KK, Mertens F (eds) WHO: Pathology and genetics of tumours of the soft tissue and bone. IARC Press, Lyon, pp 257-258

15. Milchgrub S, Hogendoorn PC (2002) Dedifferentiated chondrosarcoma. In: Fletcher CDM, Unni KK, Mertens F (eds) WHO: Pathology and genetics of tumours of the soft tissue and bone. IARC Press, Lyon, pp 252-254

16. Nakashima Y, Park Y, Sugano O (2002) Mesenchymal chondrosarcoma. In: Fletcher CDM, Unni KK, Mertens F (eds) WHO: Pathology and genetics of tumours of the soft tissue and bone. IARC Press, Lyon, pp 255-256

17. Nakashima Y, Unni KK, Shives TC et al. (1986) Mesenchymal chondrosarcoma of bone and soft tissue. Cancer 57: 2444-2453

18. Nojima T, Unni KK, McLeod RA et al. (1985) Periosteal chondroma and periosteal chondrosarcoma. Am J Surg Pathol 9: 666-677

19. Ostrowski ML, Spjut HJ, Bridge JA (2002) Chondromyxoid fibroma. In: Fletcher CDM, Unni KK, Mertens F (eds) WHO: Pathology and genetics of tumours of the soft tissue and bone. IARC Press, Lyon, pp 243-245
20. Porter DE, Lonie L, Fraser M et al. (2004) Severity of disease and risk of malignant change in hereditary multiple exostoses. A genotype-phenotype study. J Bone Joint Surg Br 86: 1041-1046

21. Romeo S, Bovee JV, Jadnanansing NA et al. (2004) Expression of cartilage growth plate signalling molecules in chondroblastoma. J Pathol 202: 113-120

22. Rozeman LB, Sangiorgi L, Briaire-de Bruijn IH et al. (2004) Enchondromatosis (Ollier disease, Maffucci syndrome) is not caused by the PTHR1 mutation p.R150C. Hum Mutat 24: 466-473

23. Sandberg AA, Bridge JA (2003) Updates on the cytogenetics and molecular genetics of bone and soft tissue tumors: chondrosarcoma and other cartilaginous neoplasms. Cancer Genet Cytogenet 143: 1-31

24. Unni KK, Inwards CY, Bridge JA et al. (2005) Tumors of the bones and joints. ARP and AFIP, Washington

25. Welkerling H, Kratz S, Ewerbeck V et al. (2003) A reproducible and simple grading system for classical chondrosarcomas. Analysis of 35 chondrosarcomas and 16 enchondromas with emphasis on recurrence rate and radiological and clinical data. Virchows Arch 443: 725-733

26. Wu CT, Inwards CY, O'Laughlin S et al. (1998) Chondromyxoid fibroma of bone: a clinicopathologic review of 278 cases. Hum Pathol 29: 438-446 